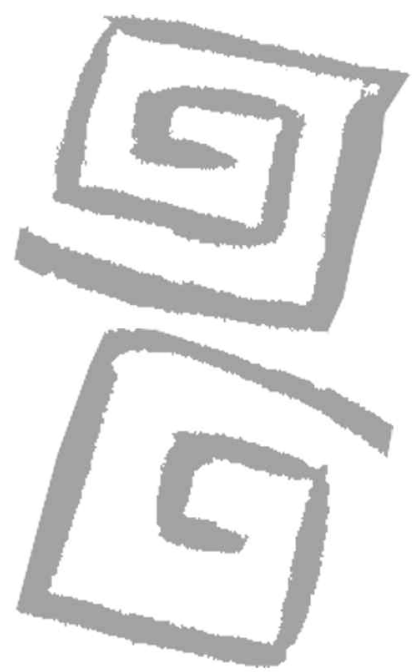

\title{
Factores predictivos de la no adherencia al tratamiento de la tuberculosis en municipios del Área Metropolitana de Buenos Aires, Argentina
}

\author{
Predictive factors of non-adherence to tuberculosis \\ treatment in the metropolitan area of Buenos Aires, \\ Argentina
}

Arrossi, Silvina1; Herrero, María Belén ${ }^{2}$; Greco, Adriana3 ; Ramos, Silvina 4

\begin{abstract}
${ }^{1}$ Socióloga. Docteur en Demographie. Investigadora adjunta, Consejo Nacional de Investigaciones Científicas y Técnicas, Centro de Estudios de Estado y Sociedad (CONICETCEDES), Argentina. silviarrossi2020@gmail.com

${ }^{2}$ Socióloga. Becaria, Consejo Nacional de Investigaciones Científicas y Técnicas, Centro de Estudios de Estado y Sociedad (CONICET-CEDES), Argentina. mbelen.herrero@gmail.com

3 Psicóloga. Magister en Ciencias Sociales y Salud. Profesional de Planta Permanente, Equipo de Salud Mental. Hospital Materno Infantil Dr. Oscar Alende, Lomas de Zamora, Argentina. aagreco@ymail.com

${ }^{4}$ Socióloga. Investigadora titular, Centro de Estudios de Estado y Sociedad (CEDES), Argentina. silvinaramosarcoiris@gmail.com
\end{abstract}

RESUMEN El objetivo de este estudio fue identificar las características sociodemográficas de los pacientes y las características del tratamiento que influyen en la no-adherencia al tratamiento antituberculoso. Se realizó un estudio de corte transversal, en el Área Metropolitana de Buenos Aires. Se encuestaron 38 pacientes que no adhirieron al tratamiento y 85 pacientes que adhirieron al tratamiento, diagnosticados durante el año 2007, residentes y atendidos en hospitales de municipios seleccionados. El análisis de los factores se llevó a cabo mediante regresión logística. Los resultados indican que los pacientes con viviendas sin agua, tuvieron 3 veces más probabilidad de no adherencia $(\mathrm{OR}=2,8 ; \mathrm{IC} 95 \%$ 1,1-6,9). Asimismo, los pacientes que realizaban los controles en un hospital tuvieron 3 veces más riesgo de no adherir que los que los realizaban en centros de atención primaria $(\mathrm{OR}=3,2 ; \mathrm{IC} 95 \%$ 1,1-8,9). Estos resultados permiten delinear un perfil de paciente en riesgo de no-adherencia, caracterizado por estar en condiciones de pobreza, y con dificultades de acceso a la atención de su salud.

PALABRAS CLAVE Tuberculosis; Tratamiento; Adhesión a la Medicación; Cooperación del Paciente; Condiciones de Vida; Atención a la Salud; Argentina.

ABSTRACT The aim of this study was to identify the socio-demographic characteristics of patients and the treatment characteristics that influence non-adherence to antituberculosis treatment. A transversal case-control study was carried out in the Metropolitan Area of Buenos Aires. Of the patients interviewed, 38 were non-adherent and 85 were adherent; all were diagnosed during 2007 and resided in and were treated in the hospitals of the selected municipalities. Factors predictive of non-adherence were assessed through logistic regression analysis. The results indicate that patients whose dwellings had no water supply were nearly 3 times more likely to be nonadherent $(\mathrm{OR}=2.8,95 \% \mathrm{Cl}$ 1.1-6.9). Patients who had medical check-ups at hospitals were 3 times more likely to be non-adherent than those with check-ups at a primary health care center $(\mathrm{OR}=3.2,95 \% \mathrm{Cl} 1.1-8.9)$. These results allow us to identify patients at risk of non-adherence to antituberculosis treatment as those living in poverty conditions and facing barriers to health care access.

KEY WORDS Tuberculosis; Treatment; Medication Adherence; Patient Compliance; Living Conditions; Health Care (Public Health); Argentina. 


\section{INTRODUCCIÓN}

Aunque la tuberculosis es curable y en general prevenible, en Argentina es uno de los principales problemas de salud pública. Cada año se diagnostican más de 10.000 nuevos casos y mueren más de 800 personas a causa de la enfermedad (1).

La no adherencia al tratamiento es considerada una de las principales barreras para el control de la enfermedad debido a las consecuencias de su interrupción -la progresión de la enfermedad y la muerte, el contagio, y el desarrollo de cepas resistentes (2)-y, en general, está asociada a la vulnerabilidad social de los pacientes. En efecto, la evidencia indica que los pacientes con peores condiciones de vida, de menor nivel socioeconómico, fundamentalmente hombres, desempleados y de mayor edad son los que menos probabilidad tienen de poder adherir al tratamiento (3-12).

En Argentina, en los últimos años, el porcentaje de los pacientes con tuberculosis bacilíferos que abandonó el tratamiento ha aumentado. En 2008, el porcentaje de abandono fue del $12 \%$, uno de los más elevados durante los últimos diez años (13).

En un esfuerzo por asegurar la adherencia al tratamiento, la comunidad médico-científica ha desarrollado la modalidad de tratamiento directamente observado (TDO), que consiste en la toma de los medicamentos antituberculosos por parte del paciente en presencia del personal de salud, en la mayoría de los casos, en el servicio de salud (14). La incorporación del TDO constituye actualmente una de las principales recomendaciones de la Organización Mundial de la Salud (OMS) para los programas de control de la tuberculosis de todo el mundo en su lucha por el control de la enfermedad. EI TDO incluye un conjunto de otras acciones dirigidas a enfrentar otros aspectos de control del problema (por ejemplo, provisión de insumos, vigilancia y monitoreo, etc.) (14).

La aplicación del TDO y el tratamiento completo de la tuberculosis son las dos intervenciones recomendadas internacionalmente para reducir el problema, y forman parte de la estrategia de tratamiento abreviado estrictamente supervisado (TAES) adoptada por Argentina. Sin embargo, si bien el Programa Nacional de Control de la
Tuberculosis recomienda la implementación del TDO, su aplicación varía en todo el territorio e incluso a nivel de los distintos servicios de salud. Esta aplicación diferencial del TDO a nivel de los servicios de salud podría tener implicancias en los resultados, en términos de adherencia al tratamiento. Una evaluación del Instituto Nacional de Enfermedades Respiratorias "Dr. Emilio Coni" relevó que, en Argentina, el 50\% de los servicios de salud no brinda acceso a la población al TDO y por lo menos 1 de cada 10 casos abandona el tratamiento antes de finalizarlo (15).

Resulta fundamental indagar los factores que influyen en la adherencia al tratamiento, teniendo en cuenta el marco específico de la implementación del TDO y su aplicación diferencial según cada servicio de salud. Según nuestro conocimiento, en nuestro país no se dispone de estudios publicados que hayan analizado de manera sistemática la condición sociodemográfica de los pacientes con tuberculosis y cómo esta se relaciona con la capacidad de adherir al tratamiento, teniendo en cuenta la modalidad de tratamiento aplicada.

El objetivo de este estudio fue analizar las características sociodemográficas de los pacientes, determinantes de la no-adherencia, en servicios de salud de municipios seleccionados del Área Metropolitana de Buenos Aires (AMBA), Argentina. El propósito es contribuir con información que pueda ser utilizada en el mejoramiento de las estrategias de control de la tuberculosis para lograr mejores resultados en la adherencia de los servicios de salud a las normas del Programa Nacional y de la población al tratamiento y cura de la enfermedad.

\section{MATERIALES Y MÉTODOS}

El estudio se llevó a cabo en municipios seleccionados de la Región Sanitaria VI del AMBA. Esta región sanitaria concentra el 13\% del total de casos notificados en el país.

Se seleccionaron siete municipios (Almirante Brown, Avellaneda, Berazategui, Esteban Echeverría, Ezeiza, Lomas de Zamora y Quilmes) en los que se identificaron los establecimientos de salud que brindan tratamiento a los 
pacientes con tuberculosis. En estos municipios la tasa de no adherencia al tratamiento en los casos pulmonares positivos, en el año 2007, fue del $13,46 \%(5,45 \%-19,16 \%)$.

\section{Población de estudio}

La población objetivo fue definida como los casos notificados con tuberculosis pulmonar, residentes en los municipios seleccionados, de 18 años o más, a los que se les hubiere prescripto tratamiento durante el año 2007 en los establecimientos de salud de dichos municipios. Se excluyeron los pacientes menores de 18 años, pacientes derivados a otro establecimiento de salud no incluido en la muestra, y los que hubieran transcurrido la mayor parte del tratamiento en situación de privación de la libertad (prisión) o con algún tipo de discapacidad intelectual, mental o física que impidiera la administración de la encuesta al paciente.

\section{Diseño del estudio}

Se realizó un estudio de corte transversal que utilizó una metodología cuantitativa para el relevamiento y análisis de la información.

A través de los registros del Programa Nacional de Control de la Tuberculosis y de la Región Sanitaria VI se identificaron a los pacientes diagnosticados con tuberculosis durante el año 2007 elegibles para participar del estudio.

En este estudio, los pacientes no adherentes fueron todos los pacientes elegibles que no adhirieron al tratamiento prescripto. De acuerdo a la definición de no adherencia de la OMS y del Programa Nacional se definió como caso no adherente aquel paciente que interrumpió el tratamiento por 60 días consecutivos o más (16-19). Los pacientes adherentes fueron los pacientes que completaron el tratamiento en forma regular (19). Tanto los no adherentes como los adherentes fueron seleccionados de la base de datos de notificación de la Región Sanitaria VI.

El protocolo del estudio fue aprobado por el Comité de Ética de cada hospital participante. El equipo de salud de cada establecimiento realizó un primer contacto telefónico con los pacientes para explicarles el estudio y confirmar los domicilios. Los pacientes fueron posteriormente entrevistados en sus domicilios por un equipo de encuestadores, mediante un cuestionario estructurado, con el fin de recolectar datos referidos a las características sociodemográficas y socioeconómicas del paciente y del hogar, como también las características del tratamiento. En el momento de la entrevista cara a cara con el encuestador se les solicitó un acuerdo de participación mediante un consentimiento informado. Se realizó una prueba piloto del cuestionario con 10 pacientes (no incluidos en el estudio) de la zona de estudio.

\section{Análisis estadístico}

Para el procesamiento y análisis de los datos se utilizó el paquete estadístico Stata/SE 9.0. Se elaboraron cuadros de frecuencias para cada una de las variables consideradas, comparando entre no adherentes y adherentes. Se calculó el test de $\mathrm{Chi}^{2}$ para evaluar la asociación entre las variables. La regresión logística univariada se utilizó para evaluar el efecto individual de cada una de las variables independientes con respecto a la adherencia o no al tratamiento. Finalmente, se realizó un análisis de regresión logística multivariada para determinar si las características sociodemográficas y la modalidad del tratamiento influyen sobre la adherencia al tratamiento, controlando el efecto de confusión potencial de estas variables. Solo las variables significativas $(p<0,05)$ en el análisis univariado fueron incorporadas en el análisis multivariado (nivel de ingresos del hogar, provisión de agua en el hogar, situación de empleo, tipo de servicio donde se realizaba los controles, tipo de servicio donde retiraba la medicación). A pesar de que la variable "tipo de servicio de dónde retiraba los medicamentos" fue significativa en el análisis univariado, no se incluyó en el modelo final dado el alto nivel de colineariedad con la variable "tipo de servicio donde realizaba los controles". Solo en el caso de dos pacientes, el lugar de retiro de la medicación y el lugar de realización de los controles no coincidió. En el análisis multivariado, un valor de $p<0,05$ se consideró como estadísticamente significativo. Se calcularon odds ratio (OR) e intervalos de confianza del 95\% (IC95\%). 
Los factores sociodemográficos que se incluyeron en el análisis (tanto para el paciente, como para el jefe de hogar) fueron: edad, sexo, nivel educativo, condición de actividad, cobertura de salud (sí/no), ingreso familiar promedio mensual (hasta 123 dólares, entre 124 y 245 dólares, más de 245 dólares), provisión de agua potable dentro la vivienda (sí/no), retrete con o sin descarga de agua (sí/no) y hacinamiento (sí/no). La ocupación se clasificó como: trabajadores con protección social, trabajadores sin protección social (incluye a los trabajadores por cuenta propia) e inactivos/desempleados. Las características del tratamiento incluidas en el análisis fueron: TDO (sí/no), tipo de establecimiento de salud donde realizaba los controles (centro de atención primaria de la salud/hospital), tipo de establecimiento de salud donde retiraba la medicación (centro de atención primaria de la salud/hospital).

\section{RESULTADOS}

En el año 2007, hubo un total de 193 casos de tuberculosis de pacientes elegibles, residentes en los municipios seleccionados y atendidos en establecimientos de salud situados en el municipio de residencia del paciente. De estos, $78(40 \%)$ no adhirieron al tratamiento antituberculoso y 115 (60\%) adhirieron al tratamiento.

En total, fueron encuestados 123 pacientes $(64 \%)$, distribuidos en 38 (31\%) no adherentes y 85 (69\%) adherentes (40 no adherentes y 30 adherentes no pudieron ser localizados, principalmente, por dificultades ligadas al registro del domicilio).

Las características sociodemográficas de los no adherentes y adherentes y sus hogares pueden observarse en el Cuadro 1 y en el Cuadro 2 , que muestran que las diferencias en las características sociodemográficas entre los no adherentes y adherentes -57 eran mujeres (46\%) y 66 eran hombres (54\%)- no fueron estadísticamente significativas. La media de edad de los no adherentes fue 37 años $(D E=2,3)$ y la de los adherentes 41 años ( $D E=1,8)$, sin diferencias estadísticamente significativas. En cuanto a la condición de actividad, la mayoría de los entrevistados se encontraba trabajando al momento del diagnóstico de tuberculosis (91\%), sin embargo solo una minoría de los pacientes y jefes de hogar ocupados tenían un empleo con protección social $(14,6 \%)$. La mayor parte de los entrevistados $(80 \%)$ no poseía cobertura de salud. Este porcentaje fue del $84 \%$ entre los no adherentes y el $78 \%$ entre los adherentes, pero las diferencias no fueron estadísticamente significativas.

Con relación a las características del tratamiento de los 123 pacientes incluidos en el estudio (Cuadro 3), el 98\% recibió tratamiento autoadministrado. Solo 2 pacientes (un no adherente y un adherente) recibieron TDO. El 68\% de los pacientes retiraba la medicación en el hospital, y el 33\% lo hacía en un centro de atención primaria, con variaciones estadísticamente significativas entre no adherentes y adherentes (el $82 \%$ y el $61 \%$ respectivamente; $p<0,05)$. Una distribución similar se observó con relación al tipo de servicio donde los pacientes realizaban los controles $(p<0,05)$.

En el Cuadro 4 se presentan los resultados del análisis univariado y multivariado. En el análisis univariado, el riesgo de no adherencia fue mayor para los pacientes de hogares cuyos ingresos mensuales no superaban los 500 pesos (equivalentes a 123 dólares) $(\mathrm{OR}=3,1$; IC95\% $1,1-8,7)$, y donde el jefe de hogar tenía un empleo sin protección social $(\mathrm{OR}=3,9$; IC95\% $0,9-18,2)$ o se encontraba inactivo o desempleado $(O R=6,7 ; \mathrm{IC} 95 \%$ 1,1-44,1). El riesgo de no adherencia al tratamiento fue mayor entre los pacientes cuyas viviendas carecían de provisión de agua en su interior, respecto de aquellos que contaban con agua en el interior de la vivienda $(\mathrm{OR}=3,1$; IC95\% 1,3-7,1). Finalmente, el riesgo de no adherencia fue mayor entre los pacientes que retiraban la medicación de un hospital, comparado con el de quienes la retiraban en un centro de atención primaria $(\mathrm{OR}=2,8$; IC95\% 1,1$7,1)$. Igualmente se observó un riesgo mayor entre aquellos que realizaban los controles en un hospital, comparado con el de quienes lo hacían en un centro de atención primaria $(\mathrm{OR}=3,7$; IC95\% 1,3-9,0).

Solo las variables significativas en el análisis univariado fueron incorporadas en el análisis multivariado (nivel de ingresos del hogar, provisión de agua en el hogar, situación de 
Cuadro 1. Características sociodemográficas de pacientes con tuberculosis (no adherentes y adherentes al tratamiento) de siete municipios de la Región Sanitaria VI. Área Metropolitana de Buenos Aires, Argentina, 2007.

\begin{tabular}{|c|c|c|c|c|c|c|c|}
\hline \multirow{2}{*}{$\begin{array}{l}\text { Características } \\
\text { sociodemográficas }\end{array}$} & \multicolumn{2}{|c|}{ No adherentes } & \multicolumn{2}{|c|}{ Adherentes } & \multicolumn{2}{|c|}{ Total } & \multirow[t]{2}{*}{ Valor de $p$} \\
\hline & $n$ & $\%$ & $n$ & $\%$ & $\mathrm{n}$ & $\%$ & \\
\hline \multicolumn{8}{|l|}{ SEXO } \\
\hline Femenino & 17 & 44,7 & 40 & 47,1 & 57 & 46,3 & \\
\hline Masculino & 21 & 55,3 & 45 & 52,9 & 66 & 53,7 & \\
\hline Total & 38 & 100,0 & 85 & 100,0 & 123 & 100,0 & NS \\
\hline \multicolumn{8}{|l|}{ EDAD } \\
\hline $18-24$ & 11 & 28,9 & 18 & 21,2 & 29 & 23,6 & \\
\hline $25-34$ & 12 & 31,6 & 32 & 37,6 & 44 & 35,9 & \\
\hline $35-64$ & 13 & 34,2 & 24 & 28,2 & 37 & 30,1 & \\
\hline 65 y más & 2 & 5,3 & 11 & 12,9 & 13 & 10,6 & \\
\hline Total & 38 & 100,0 & 85 & 100,0 & 123 & 100,0 & NS \\
\hline \multicolumn{8}{|l|}{ NIVEL EDUCATIVO } \\
\hline No asistió/primario incompleto & 8 & 21,1 & 25 & 29,4 & 33 & 26,8 & \\
\hline Primario completo & 12 & 31,6 & 20 & 23,5 & 32 & 26,0 & \\
\hline Secundario o más & 18 & 47,4 & 40 & 47,1 & 58 & 47,2 & \\
\hline Total & 38 & 100,0 & 85 & 100,0 & 123 & 100,0 & NS \\
\hline \multicolumn{8}{|l|}{ COBERTURA DE SALUD } \\
\hline No & 32 & 84,2 & 66 & 77,6 & 98 & 79,7 & \\
\hline Sí & 6 & 15,8 & 19 & 22,4 & 25 & 20,3 & \\
\hline Total & 38 & 100,0 & 85 & 100,0 & 123 & 100,0 & NS \\
\hline \multicolumn{8}{|l|}{ CONDICIÓN DE ACTIVIDAD } \\
\hline \multicolumn{8}{|l|}{ Activos } \\
\hline Ocupado & 25 & 65,8 & 58 & 68,2 & 83 & 67,5 & \\
\hline Desocupado & 4 & 10,5 & 7 & 8,2 & 11 & 8,9 & \\
\hline \multicolumn{8}{|l|}{ Inactivo } \\
\hline Ama de casa & 3 & 7,9 & 9 & 10,6 & 12 & 9,8 & \\
\hline Jubilado & 2 & 5,3 & 2 & 2,4 & 4 & 3,3 & \\
\hline Otros & 4 & 10,5 & 9 & 10,6 & 13 & 10,6 & \\
\hline Total & 38 & 100,0 & 85 & 100,0 & 123 & 100,0 & NS \\
\hline
\end{tabular}

NS = No significativo.

empleo, tipo de servicio donde se realizaba los controles, tipo de servicio donde retiraba la medicación).

En el análisis multivariado (Cuadro 4), las variables que presentaron asociación estadísticamente significativa con la no-adherencia al tratamiento fueron: tipo de provisión de agua de la vivienda y tipo de establecimiento de salud donde el paciente realizaba los controles médi- cos. Los pacientes cuyas viviendas no contaban con agua, tuvieron casi 3 veces más probabilidad de no adherencia que los que vivían en viviendas que sí contaban con agua $(\mathrm{OR}=2,8$; IC95\% 1,1$6,9)$. Asimismo, los pacientes que realizaban los controles en un hospital tuvieron 3 veces más riesgo de no adherir al tratamiento que aquellos que los realizaban en los centros de atención primaria $(\mathrm{OR}=3,2$; IC95\% 1,1-8,9). 
Cuadro 2. Características sociodemográficas de los jefes de hogar y de los hogares de pacientes con tuberculosis (no adherentes y adherentes al tratamiento) de siete municipios de la Región Sanitaria VI. Área Metropolitana de Buenos Aires, Argentina, 2007.

\begin{tabular}{|c|c|c|c|c|c|c|c|}
\hline \multirow{2}{*}{$\begin{array}{l}\text { Características } \\
\text { sociodemográficas }\end{array}$} & \multicolumn{2}{|c|}{ No adherentes } & \multicolumn{2}{|c|}{ Adherentes } & \multicolumn{2}{|c|}{ Total } & \multirow[t]{2}{*}{ Valor de $p$} \\
\hline & n & $\%$ & $\mathrm{n}$ & $\%$ & $\mathrm{n}$ & $\%$ & \\
\hline \multicolumn{8}{|l|}{ PROVISIÓN DE AGUA } \\
\hline Dentro de la vivienda & 22 & 57,9 & 68 & 81,0 & 90 & 73,8 & \\
\hline Fuera de la vivienda & 16 & 42,1 & 16 & 19,0 & 32 & 26,2 & \\
\hline Total & 38 & 100,0 & $84^{*}$ & 100,0 & 122 & 100,0 & 0,007 \\
\hline \multicolumn{8}{|l|}{ TIPO DE BAÑo } \\
\hline Retrete con descarga de agua & 21 & 55,3 & 57 & 67,1 & 78 & 63,4 & \\
\hline Retrete sin descarga de agua o letrina & 17 & 44,7 & 28 & 32,9 & 45 & 36,6 & \\
\hline Total & 38 & 100,0 & 85 & 100,0 & 123 & 100,0 & NS \\
\hline \multicolumn{8}{|l|}{ HACINAMIENTO } \\
\hline Sí & 4 & 10,5 & 6 & 7,1 & 10 & 8,1 & \\
\hline No & 38 & 89,5 & 79 & 92,9 & 113 & 91,9 & \\
\hline Total & 38 & 100,0 & 85 & 100,0 & 123 & 100,0 & NS \\
\hline \multicolumn{8}{|l|}{ NIVEL DE INGRESOS DEL HOGAR } \\
\hline Hasta 123 USD & 17 & 44,7 & 16 & 19,3 & 33 & 27,3 & \\
\hline Entre 124-245 USD & 13 & 34,2 & 44 & 53,0 & 57 & 47,1 & \\
\hline Más de 245 USD & 8 & 21,1 & 23 & 27,7 & 31 & 25,6 & \\
\hline Total & 38 & 100,0 & $83^{* *}$ & 100,0 & 121 & 100,0 & 0,014 \\
\hline \multicolumn{8}{|l|}{ COBERTURA DE SALUD DEL JEFE } \\
\hline No & 32 & 84,2 & 57 & 67,1 & 89 & 72,4 & \\
\hline Sí & 6 & 15,8 & 28 & 32,9 & 34 & 27,6 & \\
\hline Total & 38 & 100,0 & 85 & 100,0 & 123 & 100,0 & 0,049 \\
\hline \multicolumn{8}{|l|}{ CONDICIÓN DE ACTIVIDAD DEL JEFE } \\
\hline \multicolumn{8}{|l|}{ Activos } \\
\hline Ocupado & 33 & 86,8 & 78 & 92,8 & 111 & 91,0 & \\
\hline Desocupado & 2 & 5,3 & 2 & 2,4 & 4 & 3,3 & \\
\hline \multicolumn{8}{|l|}{ Inactivo } \\
\hline Ama de casa & 0 & 0 & 2 & 2,4 & 2 & 1,6 & \\
\hline Jubilado & 2 & 5,3 & 2 & 2,4 & 4 & 3,3 & \\
\hline Otros & 1 & 2,6 & 0 & 0 & 1 & 0,8 & \\
\hline Total & 38 & 100,0 & $84^{*}$ & 100,0 & 122 & 100,0 & NS \\
\hline \multicolumn{8}{|l|}{ SITUACIÓN DE EMPLEO } \\
\hline Trabajador con protección social & 2 & 5,3 & 16 & 18,8 & 18 & 14,6 & \\
\hline Trabajador sin protección social & 31 & 81,6 & 63 & 74,1 & 94 & 76,4 & \\
\hline Inactivo o desempleado & 5 & 13,2 & 6 & 7,1 & 11 & 9,0 & \\
\hline Total & 38 & 100,0 & 85 & 100,0 & 123 & 100,0 & NS \\
\hline
\end{tabular}


Cuadro 3. Características del tratamiento y de la atención de salud en pacientes con tuberculosis (no adherentes y adherentes al tratamiento) de siete municipios de la Región Sanitaria VI. Área Metropolitana de Buenos Aires, Argentina, 2007.

\begin{tabular}{|c|c|c|c|c|c|c|c|}
\hline \multirow{2}{*}{$\begin{array}{l}\text { Características del tratamiento y de la atención } \\
\text { de salud }\end{array}$} & \multicolumn{2}{|c|}{ No adherentes } & \multicolumn{2}{|c|}{ Adherentes } & \multicolumn{2}{|c|}{ Total } & \multirow[t]{2}{*}{ Valor de $p$} \\
\hline & $\mathrm{n}$ & $\%$ & $\mathrm{n}$ & $\%$ & n & $\%$ & \\
\hline \multicolumn{8}{|l|}{ MODALIDAD DE TRATAMIENTO } \\
\hline Tratamiento autoadministrado & 37 & 97,4 & 84 & 98,8 & 121 & 98,4 & \\
\hline Tratamiento directamente observado & 1 & 2,6 & 1 & 1,2 & 2 & 1,6 & \\
\hline Total & 38 & 100,0 & 85 & 100,0 & 123 & 100,0 & NS \\
\hline \multicolumn{8}{|l|}{ TIPO DE SERVICIO DONDE RETIRABA LA MEDICACIÓN } \\
\hline Hospital & 31 & 81,6 & 52 & 61,2 & 89 & 67,5 & \\
\hline Centro de Atención Primaria & 7 & 18,4 & 33 & 38,8 & 40 & 32,5 & \\
\hline Total & 38 & 100,0 & 85 & 100,0 & 123 & 100,0 & 0,026 \\
\hline \multicolumn{8}{|l|}{ TIPO DE SERVICIO DONDE REALIZABA LOS CONTROLES } \\
\hline Hospital & 31 & 81,6 & 51 & 60,0 & 82 & 66,7 & \\
\hline Centro de Atención Primaria & 7 & 18,4 & 34 & 40,0 & 41 & 33,3 & \\
\hline Total & 38 & 100,0 & 85 & 100,0 & 123 & 100,0 & 0,022 \\
\hline
\end{tabular}

\section{DISCUSIÓN}

Según nuestro conocimiento, esta es la primera vez que en Argentina se analiza de manera sistemática la condición sociodemográfica de los pacientes con tuberculosis y cómo esta incide en su capacidad de adherir al tratamiento. El principal resultado de este estudio es que en la población analizada, la provisión de agua en la vivienda de los pacientes, y el lugar en donde se realiza el control médico del tratamiento son determinantes de la adherencia. Son los pacientes que viven en viviendas sin provisión de agua, y aquellos que realizan los controles médicos del tratamiento en hospitales (en contraposición a los que lo hacen en los centros de atención primaria), los que tienen mayor riesgo de no adherir al tratamiento. Un importante hallazgo de nuestro estudio es que, si bien en Argentina se recomienda la aplicación del TDO, el 98\% de la población analizada recibió tratamiento autoadministrado, y solo 2 pacientes (un no adherente y un adherente) recibieron TDO.

En Argentina, la no provisión de agua es considerada un indicador de pobreza estructural y forma parte de uno de los servicios esenciales que debe tener una vivienda según el Instituto Nacional de Estadísticas y Censos (INDEC) (20).
Por otra parte, a la asociación encontrada en nuestro estudio entre la no provisión de agua, como indicador de pobreza, y la adherencia al tratamiento, se suma el hecho de que los pacientes con menor nivel de ingreso tuvieron una probabilidad casi dos veces mayor de no adherir al tratamiento. El hecho de que este aumento del riesgo de no adherencia para los pacientes de más bajos ingresos no haya sido estadísticamente significativo puede haberse debido al limitado tamaño de la muestra. El conjunto de estos resultados estarían indicando que las deficientes condiciones de vida de los pacientes influyen negativamente en la capacidad de adherir al tratamiento. La asociación entre nivel socioeconómico y la no adherencia al tratamiento de la tuberculosis ha sido analizada en una variedad de estudios llevados a cabo en distintos países y regiones $(5,6,21-24)$. En un estudio realizado en España en un grupo de pacientes seropositivos y con tuberculosis, se halló que residir en una zona de bajo nivel socioeconómico suponía 1,6 veces mayor riesgo de no adherencia al tratamiento (21). Para Cáceres y Orozco (23), que analizaron en Colombia una cohorte de pacientes bajo TDO, ese riesgo es aún mayor: según estos autores, los pacientes con un nivel socioeconómico bajo tienen casi 4 veces más probabilidades de abandonar el tratamiento antituberculoso que los pacientes 
Cuadro 4. Características sociodemográficas de pacientes con tuberculosis y características asociadas a la no adherencia al tratamiento. Regresión logística univariada y multivariada. Siete municipios de la Región Sanitaria VI, Área Metropolitana de Buenos Aires, Argentina, 2007.

\begin{tabular}{|c|c|c|c|c|c|c|}
\hline \multirow[t]{2}{*}{ Características sociodemográficas y del tratamiento } & \multicolumn{3}{|c|}{ Análisis univariado } & \multicolumn{3}{|c|}{ Análisis multivariado } \\
\hline & OR & IC95\% & Valor de $\mathrm{p}$ & OR & IC95\% & Valor de $p$ \\
\hline \multicolumn{7}{|l|}{ NIVEL DE INGRESOS DEL HOGAR } \\
\hline Más de 245 USD & 1 & - & - & 1 & - & - \\
\hline Entre 124-245 USD & 0,8 & $(0,3 ; 2,3)$ & NS & - & - & NS \\
\hline Hasta 123 USD & 3,1 & $(1,0 ; 8,7)$ & 0,038 & 2,4 & $(0,9 ; 8,7)$ & 0,05 \\
\hline \multicolumn{7}{|l|}{ PROVISIÓN DE AGUA EN EL HOGAR } \\
\hline Sí & 1 & - & - & 1 & - & - \\
\hline No & 3,1 & $(1,3 ; 7,1)$ & 0,009 & 2,8 & $(1,1 ; 6,9)$ & 0,031 \\
\hline \multicolumn{7}{|l|}{ COBERTURA DE SALUD DEL JEFE DE HOGAR } \\
\hline Sí & 1 & - & - & - & - & - \\
\hline No & 2,6 & $((0,9 ; 6,9)$ & 0,05 & - & - & - \\
\hline \multicolumn{7}{|l|}{ SITUACIÓN DE EMPLEO } \\
\hline Trabajador con protección social & 1 & - & - & - & - & - \\
\hline Trabajador sin protección social & 3,9 & $(0,9 ; 18,2)$ & NS & - & - & NS \\
\hline Inactivo o desempleado & 6,7 & $(1,1-44,1)$ & 0,04 & - & - & NS \\
\hline \multicolumn{7}{|l|}{ TIPO DE SERVICIO DONDE RETIRABA LA MEDICACIÓN } \\
\hline Centro de Atención Primaria & 1 & - & - & - & - & - \\
\hline Hospital & 2,8 & $(1,1 ; 7,1)$ & 0,029 & - & - & 0,5 \\
\hline \multicolumn{7}{|l|}{ TIPO DE SERVICIO DONDE REALIZABA LOS CONTROLES } \\
\hline Centro de Atención Primaria & 1 & - & - & 1 & - & - \\
\hline Hospital & 3,7 & $(1,3 ; 9,0)$ & 0,014 & 3,2 & $(1,3 ; 8,9)$ & 0,028 \\
\hline
\end{tabular}

con niveles socioeconómicos más altos. Galiano y Montesinos (24) encontraron, en un estudio llevado a cabo en Chile, que el riesgo de abandono del tratamiento es casi 2 veces mayor en los pacientes sin ingreso. En un estudio realizado en Nicaragua (6) resultaron asociados al abandono otros factores indicativos de situación socioeconómica precaria como no tener techo o sufrir frecuentes cambios de domicilio. En el estudio de Mishra et al. (5), en Nepal, los pacientes con un ingreso anual bajo tienen 5 veces más probabilidad de no adherencia al tratamiento que aquellos que tienen un ingreso medio o alto. En estos estudios, los autores hallaron que a pesar de la gratuidad del tratamiento, un bajo nivel socioeconómico influye negativamente en la adherencia debido al costo del transporte para asistir al servicio de salud y el costo en términos de ingresos por la pérdida de la jornada laboral $(5,6,20)$.
En nuestro estudio, el hecho de que el tratamiento para tuberculosis sea gratuito sugiere que factores distintos al costo del tratamiento están influyendo en una mayor no adherencia de los pacientes con bajo nivel socioeconómico. Un estudio Ilevado a cabo en Argentina (25) sobre adherencia al tratamiento de pacientes con cáncer mostró que uno de los principales factores que reducía la adherencia era la pérdida de ingresos que resulta de la interrupción de la actividad laboral durante el tratamiento cuando los trabajadores carecen de protección social. Como consecuencia de esto, el nivel de ingresos del hogar se reduce, y el paciente tiene dificultades para afrontar el costo del transporte al hospital y otros gastos asociados al tratamiento (25). Así, en contextos de alta informalidad y de bajo nivel de ingresos, sin protección social, la capacidad de cumplir con el tratamiento se reduce, ya que para 
el paciente implica la pérdida de días laborales y por ende de ingresos básicos, como asimismo lo muestra la investigación de Balasubramanian et al. (26). En nuestro estudio, el hecho de que los trabajadores sin protección social hayan tenido una probabilidad casi 4 veces mayor de no adherir al tratamiento (resultado estadísticamente no significativo), sugiere que la vulnerabilidad frente al impacto económico de la enfermedad, podría estar influyendo en la no adherencia, sobre todo en pacientes de bajo nivel socioeconómico. En el estudio de Mishra et al. (5) se destaca justamente la influencia tanto del desempleo como del empleo precario en la no-adherencia, junto con el bajo ingreso y el costo del transporte. Al respecto, los autores mencionan que estas dificultades se profundizan por el costo que implica concurrir a los centros de salud, en especial, en relación con la pérdida del ingreso laboral. Esto es coincidente con los resultados encontrados en el estudio de O’Boyle (11) con relación al tiempo empleado en el traslado para asistir al servicio de salud. Los autores mencionan que el mismo podría implicar pérdidas de tiempo de la jornada laboral, con la consecuente reducción de ingresos, lo que podría explicar el mayor número de empleados entre los no-adherentes (11).

Nuestro estudio encontró que a pesar de las recomendaciones del Programa Nacional de Control de la Tuberculosis sobre la aplicación del TDO, en los servicios de salud seleccionados la implementación del TDO es baja (2\%). Los resultados muestran que en un contexto de tratamiento mayoritariamente autoadministrado, los pacientes que retiran la medicación y que realizan los controles médicos en los hospitales (en contraposición a los que lo hacen en los centros de atención primaria), tienen mayor riesgo de no adherir al tratamiento. Esto es coincidente con los resultados de un estudio realizado en Sudán que encontró una mayor adherencia al tratamiento entre los pacientes que lo realizaron en centros de atención primaria en comparación con aqueIlos que lo realizaron en hospitales (27). La principal conclusión fue que cuanto más cerca se encuentra el centro de salud de la vivienda de los pacientes, mayor es la adherencia al tratamiento (27). Otros estudios también han mostrado que cuanto más tiene que viajar el paciente para recibir el tratamiento, menor es la probabilidad de que este pueda Ilevarlo a cabo de manera continua (11,28-31). En Argentina el centro de atención primaria constituye el primer eslabón de la cadena de atención. La importancia de estos centros radica en que su distribución en el territorio facilita el acceso de la población a los servicios de atención primaria de la salud reduciendo algunas de las barreras geográficas de acceso a la atención sanitaria $(32,33)$. La cercanía de los centros en relación con la vivienda de los pacientes podría ser entonces uno de los factores que explican la mayor adherencia entre aquellos que realizan los controles médicos en ese tipo de establecimientos de salud. Sin embargo, un hecho a destacar es que los estudios que han encontrado una relación entre adherencia y distancia al establecimiento de salud, han sido implementados en contextos de TDO, es decir, que el paciente tiene que viajar todos los días para tomar la medicación bajo la supervisión del profesional de la salud. Las dificultades ligadas a la organización de los hospitales de mayor complejidad (alta demanda, poco personal, largas esperas, atención menos personalizada) también podrían estar teniendo un rol en la menor adherencia observada en los hospitales de mayor complejidad.

Si bien consideramos fundamental analizar cómo influye el TDO en la capacidad de adherir al tratamiento en el contexto del sistema de salud en Argentina, no fue posible en nuestro estudio realizar este análisis debido al bajo porcentaje de pacientes bajo TDO (2\%). Nuestros datos muestran que en los servicios de salud seleccionados, correspondientes a una de las principales regiones sanitarias de la provincia de Buenos Aires, no se siguen completamente las recomendaciones nacionales respecto de la aplicación del TDO (34). Los resultados de este estudio indican que debieran analizarse cuáles son los obstáculos que impiden la implementación programática del TDO en estos servicios.

Consideramos que este estudio tiene algunas limitaciones metodológicas que son importantes mencionar. En primer lugar nuestro estudio puede estar afectado por una tasa de no respuesta mayor en los no adherentes que en los adherentes, lo que implicaría un sesgo de selección. En efecto, el $51 \%$ de los casos y el $26 \%$ de los controles no pudieron ser entrevistados debido a problemas ligados a la falta de datos del 
Cuadro 5. Comparación de las características sociodemográficas de pacientes con tuberculosis (no adherentes y adherentes) según participación en el estudio. Siete municipios de la Región Sanitaria VI, Área Metropolitana de Buenos Aires, Argentina, 2007.

\begin{tabular}{|c|c|c|c|c|c|}
\hline \multirow{2}{*}{$\begin{array}{l}\text { Características } \\
\text { sociodemográficas }\end{array}$} & \multicolumn{2}{|c|}{ No adherentes $(n=78)$} & \multicolumn{2}{|c|}{ Adherentes $(n=115)$} & \multirow[t]{2}{*}{ Valor de $p$} \\
\hline & $\begin{array}{c}\text { Encuestado } \\
\quad(n=38)\end{array}$ & $\begin{array}{c}\text { No encuestado } \\
(n=40)\end{array}$ & $\begin{array}{l}\text { Encuestado } \\
\quad(n=85)\end{array}$ & $\begin{array}{c}\text { No encuestado } \\
(n=30)\end{array}$ & \\
\hline Media de edad (desviación estándar) & $37(2,3)$ & $36(2,5)$ & $41(1,8)$ & $35(2,3)$ & NS \\
\hline \multicolumn{6}{|l|}{ SEXO } \\
\hline Femenino & $31,6 \%$ & $25,0 \%$ & $50,6 \%$ & $60,0 \%$ & NS \\
\hline Masculino & $68,4 \%$ & $75,0 \%$ & $49,4 \%$ & $40,0 \%$ & - \\
\hline
\end{tabular}

domicilio y el $17 \%$ de los pacientes no pudieron ser encontrados ni personal ni telefónicamente. Esto es coincidente con otros estudios que también han mostrado una mayor dificultad para encontrar a los pacientes que no adhirieron al tratamiento $(11,29)$. En nuestro estudio, se realizó una comparación de las características sociodemográficas de los adherentes y no adherentes, según la participación en el estudio y las diferencias no fueron significativas (Cuadro 5).

Por otra parte, si bien fueron entrevistados el $64 \%(n=123)$ de los pacientes elegibles $(n=193)$, la población de pacientes elegibles de nuestro estudio constituye solo el $32 \%$ de la totalidad de pacientes con tuberculosis en dichos municipios. Esto se explica por el alto porcentaje de pacientes que reciben tratamiento en hospitales ubicados en otras jurisdicciones del AMBA. Por lo tanto, nuestros resultados no son generalizables a la totalidad de los pacientes con tuberculosis de los municipios seleccionados. Finalmente, solo un paciente no aceptó participar en nuestro estudio, lo que resultó en una tasa de participación cercana al 100\%.

Consideramos que nuestro estudio contribuye a la identificación de factores relativos a las características sociodemográficos de los pacientes que influyen en la adherencia al tratamiento. Como conclusión, nuestros resultados sugieren que la descentralización del tratamiento al primer nivel de atención contribuiría a aumentar la capacidad de adherencia de los pacientes, probablemente al reducir las barreras de acceso a los servicios de salud donde se llevan a cabo el tratamiento y los controles. La protección social de los pacientes más vulnerables debiera considerarse prioritaria dentro de las estrategias de control de la enfermedad, de manera de reducir los obstáculos a la adherencia.

\section{AGRADECIMIENTOS}

Agradecemos a la Comisión Nacional Salud, Ciencia y Tecnología del Ministerio de Salud de la Nación, por apoyar este proyecto y brindar los recursos necesarios para poder Ilevarlo adelante (Estudio Colaborativo Multicéntrico "Evaluación de los factores predictivos de la no adherencia al tratamiento de tuberculosis en municipios seleccionados del Área Metropolitana de Buenos Aires"); a la Responsable Regional del Programa de Control de Tuberculosis de la Región Sanitaria VI; a Karina Faccia por su participación en la organización del trabajo de campo; a Richard Muwonge por su colaboración y sus aportes en el diseño metodológico del estudio; al personal de los hospitales incluidos en el estudio; al equipo de encuestadores y, en particular, a su coordinadora por el compromiso con la tarea y esfuerzo realizado para la búsqueda de los pacientes; al responsable del diseño y armado de la base de datos del estudio; a las/os trabajadoras/es sociales que colaboraron con el trabajo de campo y nos ayudaron a contactar a los entrevistados. Y agradecemos fundamentalmente a cada uno de los pacientes que participaron de este estudio y que compartieron sus historias para hacer posible los resultados que aquí se presentan. 


\section{REFERENCIAS BIBLIOGRÁFICAS}

1. Instituto Nacional de Enfermedades Respiratorias "Dr. Emilio Coni". Notificación de casos de tuberculosis en la República Argentina: Período 19802009. Buenos Aires: Ministerio de Salud, Presidencia de la Nación; 2010.

2. Center for Diseases Control. Core curriculum on tuberculosis: What the clinician should know. 4th ed. Atlanta: US Department of Health \& Human Services; 2000.

3. Comolet $\mathrm{T}$, Rakotomalala R, Rajaonarioa $\mathrm{H}$. Factors determining compliance with tuberculosis treatment in an urban environment, Tamatave, Madagascar. International Journal of Tuberculosis and Lung Disease. 1998;2(11):891-897.

4. Kaona F, Tuba M, Siziya S, Sikaona L. An assessment of factors contributing to treatment adherence and knowledge of TB transmission among patients on TB treatment. BMC Public Health [Internet]. 2004;4(68) [citado 12 ago 2012]. Disponible en: http://www.ncbi.nlm. nih.gov/pmc/articles/PMC545081/.

5. Mishra P, Hansen E, Sabroe S, Kafle K. Socioeconomic status and adherence to tuberculosis treatment: a case-control study in a district of Nepal. International Journal of Tuberculosis and Lung Disease. 2005;9(10):1134-1139.

6. Sosa Pineda N, Pereira S, Barreto M. Abandono del tratamiento de la tuberculosis en Nicaragua: resultados de un estudio comparativo. Revista Panamericana de Salud Pública. 2005;17(4):271278.

7. Shargie E, Lindtjørn B. Determinants of treatment adherence among smear-positive pulmonary tuberculosis patients in southern Ethiopia. PLoS Medicine [Internet]. 2007;4(2) [citado 12 ago 2012]. Disponible en: http://www.plosmedicine.org/article/info \%3Adoi \%2F10.1371\%2Fjou rnal.pmed.0040037.

8. Hasker E, Khodjikhanov M, Usarova S, Asamidinov $U$, Yuldashova $U$, van der Werf MJ, Uzakova G, Veen J. Default from tuberculosis treatment in Tashkent, Uzbekistan; Who are these defaulters and why do they default? BMC Infectious Diseases [Internet]. 2008 [citado 12 ago 2012];8(97). Disponible en: http://www.ncbi.nlm.nih.gov/pmc/articles/PM C2492865/.

9. Sumartojo E. When tuberculosis treatment fails: A social behavioral account of patient adhe- rence. American Review of Respiratory Disease. 2003;147(5):1311-1320.

10. Farmer $P$. Social scientists and the new tuberculosis. Social Science \& Medicine. 1997;44(3):347-358.

11. O'Boyle S, Power J, Ibrahim M, Watson J. Factors affecting patient compliance with antituberculosis chemotherapy using the directly observed treatment short-course strategy (DOTS). International Journal of Tuberculosis and Lung Disease. 2002;6(4):307-312.

12. Singh V, Jaiswal A, Porter J, Ogden J, Sarin R, Sharma PP, Arora VK, Jain RC. TB control, poverty, and vulnerability in Delhi, India. Tropical Medicine \& International Health. 2002; 7(8):693-700.

13. Instituto Nacional de Enfermedades Respiratorias "Dr. Emilio Coni". Resultado del tratamiento de la tuberculosis pulmonar ED (+) en la República Argentina. Período 1980-2009. Buenos Aires: Ministerio de Salud, Presidencia de la Nación; 2010.

14. World Health Organization. The stop TB strategy: Building on and enhancing DOTS to meet the TB-related millennium development goals (WHO/HTM/TB/2006.368). Geneva: WHO; 2006.

15. Instituto Nacional de Enfermedades Respiratorias "Dr. Emilio Coni". Evaluación de tratamiento de los casos de tuberculosis pulmonar Examen Directo positivo en la República Argentina. Buenos Aires: Ministerio de Salud, Presidencia de la Nación; 2006.

16. World Health Organization. WHO Tuberculosis Programme: Framework for effective Tuberculosis control (WHO/TB/94.179). Geneva: WHO; 1994.

17. World Health Organization. Treatment of tuberculosis: guidelines for national programmes (WHO/CDS/TB/2003.313). 3th ed. Geneva: WHO; 2003.

18. World Health Organization. WHO Report 2007 Global tuberculosis control: surveillance, planning, financing (WHO/HTM/TB/2007.376). Geneva: WHO; 2007.

19. Zerbini EV, Darnaud RMH, Prieto VG. Programa Nacional de Control de la Tuberculosis: Normas Técnicas 2008. 3ra ed. Santa Fe: Instituto Nacional de Enfermedades Respiratorias “Dr. Emilio Coni"; 2008. 
20. Instituto Nacional de Estadísticas y Censos. Vivienda, hogares y hábitat [Internet]. Buenos Aires: INDEC [citado 12 ago 2012]. Disponible en: http://www.indec.gov.ar/principal.asp?id_tema $=75$

21. Galdós-Tangüis H, Caylá JA, García de Olalla P, Jansá JM, Brugal MT. Factors predicting noncompletion of tuberculosis treatment among HIVinfected patients in Barcelona (1987-1996). International Journal of Tuberculosis and Lung Disease. 2000;4(1):55-60.

22. Culqui D, Grijalva C, Reategui S, Cajo J, Suárez L. Factores pronósticos del abandono del tratamiento antituberculoso en una región endémica del Perú. Revista Panamericana de Salud Pública. 2005;18(1):14-20.

23. Cáceres F, Orozco L. Incidencia y factores asociados al abandono del tratamiento antituberculoso. Revista Biomédica. 2007;27(4):498-504.

24. Galiano M, Montesinos N. Modelo predictivo de abandono del tratamiento antituberculoso para la región Metropolitana de Chile. Enfermería Clínica. 2005;15(4):192-198.

25. Arrossi S, Matos E, Zengarini N, Roth B, Sankaranarayanan R, Parkin M. The socio-economic impact of cervical cancer on patients and their families in Argentina, and its influence on radiotherapy compliance: Results from a crosssectional study. Gynaecologic Oncology. 2007;105(2):335-340.

26. Balasubramanian R, Garg R, Santha T, Gopi PG, Subramani R, Chandrasekaran V, Thomas A, Rajeswari R, Anandakrishnan S, Perumal M, Niruparani C, Sudha G, Jaggarajamma K, Frieden TR, Narayanan PR. Gender disparities in tuberculosis: report from a rural DOTS programme in south India. International Journal of Tuberculosis and Lung Disease. 2004;8(3):323-332.

27. El-Sony A, Mustafa S, Khamis A, Enarson D, Baraka O, Bjune $\mathrm{G}$. The effect of decentralization on tuberculosis services in three states of Sudan. International Journal of Tuberculosis and Lung Disease. 2003;7(5):445-450.

28. Farmer $\mathrm{P}$, Robin S, Ramilus SL, Kim J. Tuberculosis, poverty, and "compliance": lessons from rural Haiti. Seminars in Respiratory Infections. 1991;6(4):373-379.

29. Naing N, D'Este C, Isa A, Salleh R, Bakar N, Mamad M. Factors contributing to poor compliance with anti-TB treatment among tuberculosis patients. Southeast Asian Journal of Tropical Medicine and Public Health. 2001;32(2):369-382.

30. Thomas C. A literature review of the problems of delayed presentation for treatment and non-completion of treatment for tuberculosis in less developed countries and ways of addressing these problems using particular implementations of the DOTS strategy. Journal of Management in Medicine. 2002;16(4-5):371-400.

31. Mateus-Solarte J, Carvajal-Barona R. Factors predictive of adherence to tuberculosis treatment: Valle del Cauca, Colombia. International Journal of Lung Disease. 2008;12(5):520-526.

32. Maceira D, Olaviaga $S$, Kremer $P$, Cejas C. Centros de Atención Primaria de la Salud: radiografía de su distribución en la Argentina (Serie Políticas Públicas Análisis No 30). Buenos Aires: CIPPEC; 2006.

33. Bascolo E, Yavich N, Urquía M. Capacidades locales de gestión como factor predictivo de la utilización y accesibilidad a los servicios de primer nivel para la obtención de cuidados prenatales. Gerencia y Políticas de Salud. 2006;6(12):119-195.

34. Ministerio de Salud de La Nación. Programa Nacional de Control de la Tuberculosis: normas técnicas 2009. 3ra ed. Buenos Aires: Ministerio de Salud; 2009.

\section{FORMA DE CITAR}

Arrossi S, Herrero MB, Greco A, Ramos S. Factores predictivos de la no adherencia al tratamiento de la tuberculosis en municipios del Área Metropolitana de Buenos Aires, Argentina. Salud Colectiva. 2012;8(Supl 1):S65-S76.

Recibido el 25 de julio de 2012

Versión final presentada el 11 de septiembre de 2012

Aprobado el 3 de octubre de 2012 